\title{
High-Frequency Calibration of Piezoelectric Displacement Sensors Using Elastic Waves Induced by Light Pressure
}

\author{
Jernej Laloš - Tomaž Požar - Janez Možina \\ University of Ljubljana, Faculty of Mechanical Engineering, Slovenia
}

In the study of ultrasound propagation in matter, displacement sensors are indispensable and of these, the most sensitive are piezoelectric sensors. In order to eliminate the intrinsic effects of the sensor from the measurements, the sensor has to be properly calibrated, which means that its transfer function has to be evaluated from a known sensor input signal and a measured sensor output signal. This has usually been done by comparing the sensor response signal to a known input signal, namely, an ultrasonic waveform, which can be theoretically calculated using mathematical models and numerical algorithms. Until now, the point-source-point-sensor model has been primarily used, while ultrasonic waves were induced mechanically either by a dropped ball or a capillary fracture. In this paper, a real-source-real-sensor model is presented. It provides a more faithful waveform construction and it enables the removal of the aperture effect from the calculated sensor transfer function, thus giving correct and universal sensor response characteristics. This was corroborated by high-frequency calibration measurements of the output signal of a Glaser-type conical sensor in two positions on both surfaces of a glass plate, while ultrasonic waves were induced by the radiation pressure of a nanosecond laser pulse.

Keywords: absolute sensor calibration, piezoelectric sensor, laser pulse, aperture effect, elastic waves in a plate, optodynamics, Green's function

Highlights

- An absolute, high-frequency calibration method of a Glaser-type piezoelectric displacement sensor.

- A way of incorporating the real dimensions of the sensor contact area and the stimulant signal origin area.

- Elimination of the sensor aperture effect and the source force distribution effect from the calculated transfer function.

- Detailed separation of spectral characteristics by their individual contributors.

\section{INTRODUCTION}

Displacement measurements are indispensable in the study of ultrasound propagation in matter. Such measurements may be carried out using sensors that operate through transduction mechanisms based on piezoelectric, electrostatic, electromagnetic or optical (mainly light interferometric) principles [1]. Of these, piezoelectric sensors are the most sensitive [2], making them especially applicable for low-amplitude measurements $(\sim 1 \mathrm{pm})$.

Piezoelectric sensors are being used in mechanical engineering, as well as for scientific and technological research. They are used for acoustic emission testing [3] and [4] and nondestructive examination of materials [4] and [5], in microseismology [6] and [7], wave propagation studies [8], light-matter interaction studies [9] and [10], optodynamics [11], and many other applications.

For the measurements to be accurate, it is essential that the sensor is properly calibrated [12]. Its transfer function has to be known so that the sensor's intrinsic effects can be removed from its output signal and displacement measurements exclusively obtained.
Sensor calibration should be carried out uniquely for the material on which the sensor is intended to perform future measurements. While each material has its specific acoustic impedance, an equal mechanical disturbance may produce a different sensor displacement due to their impedance mismatch.

Such calibrations have usually been carried out by comparing the response of the sensor under consideration to a known stimulant signal, namely, a surface waveform, which has been either measured by another, precalibrated sensor or calculated theoretically [12] and [13]. With the use of a precalibrated sensor, both calibrations have to be carried out using the same materials, the same sensor apertures and at the same relative positions. While this is also true for the theoretical calculation, the latter is more universal and less laborious as the parameters are easily changed in the model. Until now, the theoretical waveform calculation has been mostly carried out using mathematical approximations which consider the sensor contact area and the stimulant signal origin area to be mere points and not of real size. This was done, because such approximations were valid as the frequencies of interest were sufficiently low. For the stimulant waveform origin, a dropped ball 
[13] and [14], a glass capillary fracture [13] and [15] or a pencil lead break [5] has usually been used [1], and [3]. In these cases, the contact times are rather long (about $1 \mu$ s for a dropped ball and $200 \mathrm{~ns}$ for a capillary fracture) and their force distributions, while concentrated in a relatively small impact area (with an estimated order of magnitude of about $10 \mu \mathrm{m}$ to 100 $\mu \mathrm{m}[16]$ ), may not be uniform over time and both may vary slightly with each repetition. All of this makes such calibrations credible only for signal wavelengths larger than the sizes of the signal origin and the sensor contact areas, which, quite undesirably, caps the calibration frequencies and results in inaccurate spectral transfer functions. Thus, the sensor aperture effect and the source force distribution effect are not addressed entirely as they are mostly avoided [13].

In this paper, an absolute high-frequency calibration of the piezoelectric Glaser-type conical sensor [1] and [3] is presented. An absolute calibration is possible, because such sensors operate without resonance and have the flattest response function among piezoelectric sensors [1]. Although this technique follows the general guidelines presented in the standard [12] and in the paper by McLaskey and Glaser [13], it improves upon them with several new features: an optodynamic interaction as the source force of the stimulant ultrasonic signal with an expanded mathematical model that incorporates real dimensions of the sensor contact area and the source force impact area and can allocate the signal's spectral characteristics to each individual contributor.

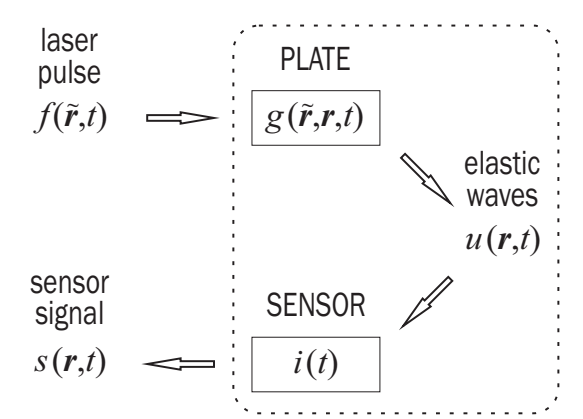

Fig. 1. Schematics of mathematical transition from the source force impulse through the plate transfer function, surface waveform and sensor transfer function to the sensor voltage output signal

The main advantage of using a laser pulse to induce the stimulant signal is that it has a very short temporal distribution and a known spatial intensity profile - both of which are independent from each other - which produces a well-determined force impulse while being consistently repeatable. The use of a laser pulse also enables a controlled size variation of the source force impact area, which in turn allows variation in the stimulant signal frequency range. In this calibration, for example, the size of the impact area is relatively large in order to demonstrate the incorporation of the macroscopic spatial extent of the source in the expanded model. It can be, however, reduced to only a few wavelengths of laser light, which is about an order of magnitude less than from any known mechanical device, thus approximating a $\delta$-source as closely as possible. Such concentration of laser light has to be used with caution, though, as the high fluence may exceed the laser-induced-damage threshold and the specimen, along with any surface coating on it, may become damaged.

The expanded mathematical model enables the proper stimulant waveform to be constructed and the sensor aperture effect [17] to be correctly accounted for, resulting in a proper and more accurate sensor spectral transfer function. The sensor can thus be correctly calibrated, even for higher frequencies than before, and used for measurement and identification of the individual wave-arrivals of ultrasonic waves in acoustic emission and laser ultrasound.

\section{METHOD}

In order for a sensor to be considered calibrated, the spectral characteristics of its transfer function have to be known. The essence of this method is, therefore, to theoretically calculate the displacement waveform that is detected by the sensor and compare it to the measured sensor output, as outlined in the standard [12] and in paper [13].

Due to the complicated nature of wave propagation in matter [8] and sensor transductivity mechanisms [15], it is convenient to introduce certain simplifications and idealizations to their mathematical description, such as the transfer function concept and the Green's function formalism.

Thus the plate and the sensor are each considered to have their own transfer functions, which transform a certain time $t$ dependent input signal into a certain different output signal. Therefore, the plate has a transfer function $g(\tilde{\boldsymbol{r}}, \boldsymbol{r}, t)$ which transforms an input source force signal $f(\tilde{\boldsymbol{r}}, t)$ at a position $\tilde{\boldsymbol{r}}$ into an output displacement signal $u(r, t)$ at another position $\boldsymbol{r}$. Similarly, the sensor has a transfer function $i(t)$ which transforms an input displacement signal $u(r, t)$ to an output voltage signal $s(\boldsymbol{r}, t)$. The schematics of this linear transform chain are shown in Fig. 1.

Both transfer functions are considered to be algebraically linear and time invariant. This is 
significant as it allows for the output signal to be expressed as a time convolution of the input signal and the appropriate transfer function:

$$
\begin{gathered}
u(\boldsymbol{r}, t)=f(\tilde{\boldsymbol{r}}, t) * g(\tilde{\boldsymbol{r}}, \boldsymbol{r}, t), \\
s(\boldsymbol{r}, t)=u(\boldsymbol{r}, t) * i(t) .
\end{gathered}
$$

Since $s(\boldsymbol{r}, t)$ is measured and $u(\boldsymbol{r}, t)$ is theoretically calculated, it is useful to perform a Fourier transform deconvolution when searching for $i(t)$. Fourier transform of Eq. (2) gives:

$$
S(\boldsymbol{r}, \omega)=U(\boldsymbol{r}, \omega) I(\omega) .
$$

To obtain the spectrum of the sensor transfer function $I(\omega)$, one must simply divide the other two transforms:

$$
I(\omega)=\frac{S(\boldsymbol{r}, \omega)}{U(\boldsymbol{r}, \omega)} .
$$

This is permissible because $U(\boldsymbol{r}, \omega)$ is not zero at any frequency $\omega \equiv 2 \pi v$ where it is defined.

The plate transfer function $g(\tilde{\boldsymbol{r}}, \boldsymbol{r}, t)$ is a superposition of individually weighted Green's functions based upon the distance distribution between points in the source force area and the sensor contact area on the plate's surface. Green's functions, in general, are the solutions of wave equations for a $\delta$-function source impulse and are highly specific for each material, shape and distance.

Each Green's function here is calculated using the modified numerical algorithm developed by Hsu [18]. It calculates Green's functions in the infinite planeparallel plate approximation, which means that it accounts for the direct waves and the waves multiple reflected from the top or the bottom surface of the plate but not for those reflected from the sides of the finite plate.

Four distance distributions and four plate transfer functions are used and compared in the calibration discussion further on. One is a simple point-sourcepoint-sensor (PP) model $g_{\mathrm{PP}}(\tilde{\boldsymbol{r}}, \boldsymbol{r}, t)$ where both the source force and the sensor are considered to act upon and from only one point on plate's surface - such as it has been known and used until now [13]. The realsource-real-sensor (RR) model $g_{\mathrm{RR}}(\tilde{\boldsymbol{r}}, \boldsymbol{r}, t)$ takes faithfully into account real dimensions of the source force impact area and the sensor contact area. The third and the fourth one are the point-source-realsensor (PR) model $g_{\mathrm{PR}}(\tilde{\boldsymbol{r}}, \boldsymbol{r}, t)$ and the real-sourcepoint-sensor (RP) model $g_{\mathrm{RP}}(\tilde{\boldsymbol{r}}, \boldsymbol{r}, t)$, which are in between the former two and are used to garner insight into the spectral characteristics and their contributors. Each of these plate transfer functions, of course, produces its own waveform model and from each of those a different sensor transfer function is calculated.

Since the arrangement is such that the source force acts only normally on the plate's surface and the sensor is assumed to detect only out-of-plane displacements, only the $z$ direction is of concern and only the $g_{z z}$ component of the elastodynamic Green's tensor is used (this direction notation, however, is omitted for simplicity).

\section{SENSOR CALIBRATION}

The sensor used here is the piezoelectric Glaser-type conical sensor: 'SteveCo' KRNBB-PC Point Contact Sensor, produced by KRN Services, Inc. [19]. This sensor is designed to detect small-scale, absolute outof-plane displacements.

The detection element in this kind of a sensor is a piezoelectric crystal PZT-5A in the shape of a truncated cone covered with a nickel electrode and backed by a heavy brass mass of irregular shape surrounded by rubber and encased in a steel case [1]. The sensor is assumed to have a uniform sensitivity over its contact area, which is circular in shape with a radius of $r_{\mathrm{S} 0}=0.5 \mathrm{~mm}$. The spatial distribution of the waveform under the sensor's aperture it therefore averaged out and combined into one output signal by the sensor. The geometric properties and electrical wiring of the sensor are specifically designed to minimize the ultrasonic and electromagnetic distorting effects. In this paper, all electrical support equipment, such as amplifiers, cables, and oscilloscope, are considered as part of the sensor.

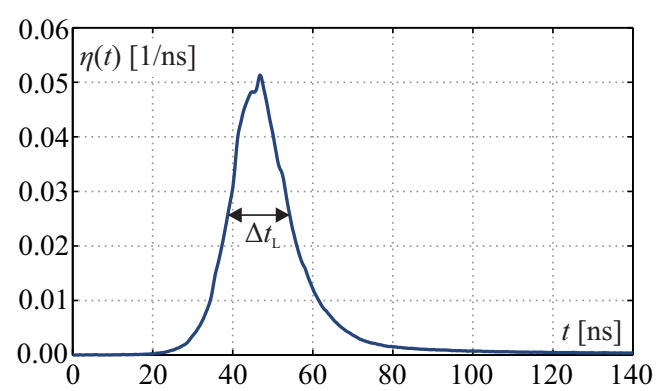

Fig. 2. Normalized temporal distribution of the incident laser pulse

The source force $f(\tilde{\boldsymbol{r}}, t)$ is the result of a laser pulse light pressure on the highly reflective mirror surface [9] and [10]. The laser pulse is produced by $\mathrm{Nd}$ :YAG laser of wavelength $\lambda_{\mathrm{L}}=1064 \mathrm{~nm}$ and energy $E_{\mathrm{L}}=200 \mathrm{~mJ}$. The pulse, which was measured with a fast photodiode, has a very short temporal distribution $\eta(t)$ with a full-width-at-half-maximum of $\Delta t_{\mathrm{L}}=17 \mathrm{~ns}$, as shown in Fig. 2. It is circularly symmetric and has a 
top-hat spatial profile [10] with a radius of $r_{\mathrm{L} 0}=1.75 \mathrm{~mm}$ on the plate's surface.

The substrate of the mirror is a plane-parallel disk-shaped glass plate made of UV-grade fused silica $\left(\mathrm{SiO}_{2}\right)$. It has a diameter of $d=50 \mathrm{~mm}$ and a thickness of $h=12 \mathrm{~mm}$. Its elastic properties are: mass density $\rho=2200 \mathrm{~kg} / \mathrm{m}^{3}$, Young's modulus $Y=72 \mathrm{GPa}$ and Poisson's ratio $\mu=0.17$. The top side of the plate is coated with a highly reflective (HR) layer with reflectivity of $R_{\mathrm{HR}}>99.8 \%$ at wavelength $\lambda_{\mathrm{L}}$. This layer does not affect the reflection of the mechanical waves as its thickness is much smaller than the wavelength of even the shortest detected waves.

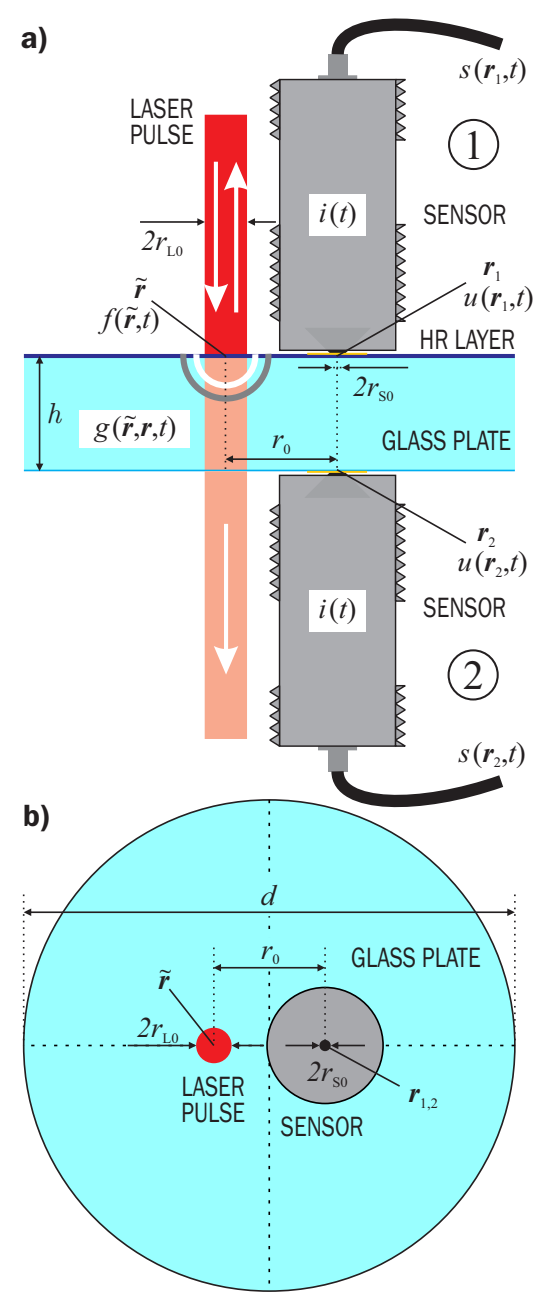

Fig. 3. Experimental setup schematic showing both positions of the piezoelectric sensor relative to the laser pulse as well as the most significant geometric properties in a) side view and in b) top view

The calibration arrangement is shown in Fig. 3. The laser pulse is incident on the top of the plate and is mostly reflected by the HR layer. The small amount of light that does pass through the plate is absorbed only insignificantly. In this manner, the pulse delivers a force impulse of $J=2 E_{\mathrm{L}} / c_{0}=1.33 \mathrm{nNs}$ normally to the plate's surface; where $c_{0}$ is the speed of light in vacuum. This impulse, in turn, generates elastic waves that propagate through the plate. These waves are therefore only light-pressure-induced and not thermoelastic or ablation-induced at all [9].

The sensor is deployed at two different positions during the calibration process. In position 1 , at $\boldsymbol{r}_{1}$, it is placed on the top surface of the plate next to the laser pulse impact area at $\tilde{\boldsymbol{r}}$ with their respective centers $r_{0}=11.5 \mathrm{~mm}$ apart. In position 2 , at $\boldsymbol{r}_{2}$, the sensor is placed on the opposite side of the plate, directly beneath position 1 .

In such an arrangement, waves reflected from the sides of the plate may reach the sensor as well: the earliest after about $8.0 \mu \mathrm{s}$ and the others well after $9.0 \mu \mathrm{s}$. It was experimentally found that the first one, the surface skimming P-wave, has such a low amplitude that it can be disregarded in this case. Therefore, for all practical purposes, the plate can be considered as infinitely large in the time period of at least the first $9.0 \mu \mathrm{s}$ after the laser pulse illumination.

Even a small amount of absorbed light by the PZT element greatly disturbs the elastic wave displacement measurement. For that reason, the sensor cannot be placed directly in the path of the laser pulse. To additionally minimize light absorption by the PZT element, a $20-\mu \mathrm{m}$-thick gold foil with some couplant was inserted between the sensor and the plate. This was done because gold has higher reflectivity $\left(\mathrm{R}_{\mathrm{Au}}=99 \%\right.$ [20] and [21]) at laser light wavelength $\lambda_{\mathrm{L}}$ than the nickel $\left(\mathrm{R}_{\mathrm{Ni}}=73 \%\right.$ [20] and [21]) that covers the sensor's tip.

The calibration measurements of the sensor output signal are averaged out of $N=200$ repetitions in each of the two sensor positions to reduce the stochastic noise and improve the signal-to-noise ratio. Each measurement has its start time set to zero by a photodiode with a rise time of $1 \mathrm{~ns}$, which is triggered when the laser pulse illuminates the plate's surface. The sampling period is $\delta t=2 \mathrm{~ns}$ while an individual measurement lasts $t_{\Omega}=9.0 \mu \mathrm{s}$, thus ending well before any significant waves reflected from the sides of the plate reach the sensor.

Since the waveform amplitudes are small, it is essencial to reduce the noise in the measurements and thus improve the signal-to-noise ratio. To illustrate the importance of this, the absolute values of the fast Fourier transform (FFT) spectra of the sensor outpust signals for both sensor positions, $\left|S_{r}\left(r_{1}, v\right)\right|$ and $\left|S_{r}\left(r_{2}, v\right)\right|$, averaged out over 200 
repeated measurements, and the absolute values of the FFT spectra of the noise in one measurement $\left|\Sigma_{1}(v)\right|$ and the noise averaged out over 200 repeated measurements $\left|\Sigma_{200}(v)\right|$, are shown in Fig. 4. The noise measurements were carried out under the same circumstances as the signal measurements except that, obviously, no stimulant force was introduced.

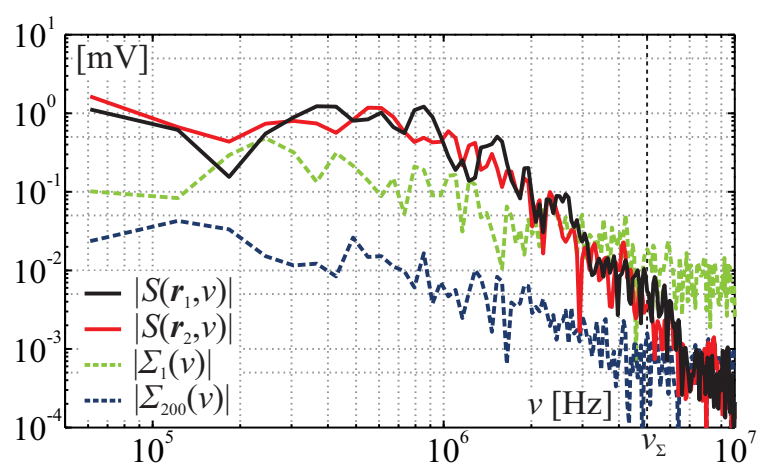

Fig. 4. A spectral comparison between the absolute values of the FFTs of the sensor output signal in both sensor positions and the sensor output noise averaged over 200 measurements and another measured only once

Comparing both noise spectra in Fig. 4, it is evident that averaging does, in fact, reduce the noise by about a decade throughout most of the frequency range. This seems consistent with signal processing theory which states that when averaging the signal over $N$ repeated measurements, its stochastic noise sould decrease by a factor of $\sqrt{N}$.

The figure also shows that the useful sensor response signal for both sensor positions is well above the averaged noise levels for frequencies smaller than about $v_{\Sigma}=5 \mathrm{MHz}$. At frequencies greater than $v_{\Sigma}$, however, the signal decreases below the averaged sensor output noise and becomes unsuitable for further processing. Any sensor calibration measurement must therefore be regarded as inaccurate at frequencies above $v_{\Sigma}$ and cannot be used for credible sensor calibration at those frequencies.

It has to be noted here that much of the output noise is most likely due to the electronics of both amplifications stages of the sensor as they were not optimized for such high-frequency measurements. The signal-to-noise ratio could be improved even more with larger laser-pulse energies and with more numerous measurement repetitions.

The sensor output measurements and calculated input waveforms are presented in Figs. 5 and 6.

Fig. 5a shows a comparison of the two theoretically calculated sensor input waveform models in picometres: a PP model $u_{\mathrm{PP}}\left(\boldsymbol{r}_{1}, t\right)$ calculated from
$g_{\mathrm{PP}}\left(\tilde{\boldsymbol{r}}, \boldsymbol{r}_{1}, t\right)$ and an RR model $u_{\mathrm{RR}}\left(\boldsymbol{r}_{1}, t\right)$ calculated from $g_{\mathrm{RR}}\left(\tilde{\boldsymbol{r}}, \boldsymbol{r}_{1}, t\right)$, with the measured sensor output voltage $s\left(\boldsymbol{r}_{1}, t\right)$ in millivolts for the sensor in position 1. In addition, Fig. $5 \mathrm{~b}$ shows a spectral comparison of the absolute values of their respective FFTs $\left|U_{\mathrm{PP}}\left(\boldsymbol{r}_{1}, v\right)\right|,\left|U_{\mathrm{RR}}\left(\boldsymbol{r}_{1}, v\right)\right|$ and $\left|S\left(\boldsymbol{r}_{1}, v\right)\right|$.

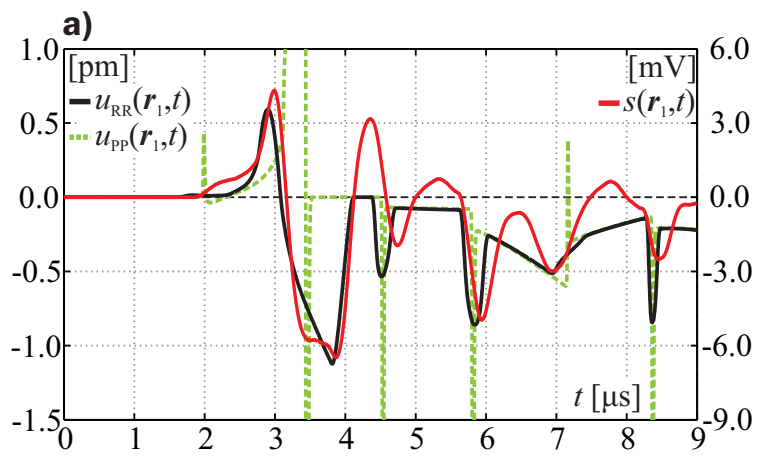

b)

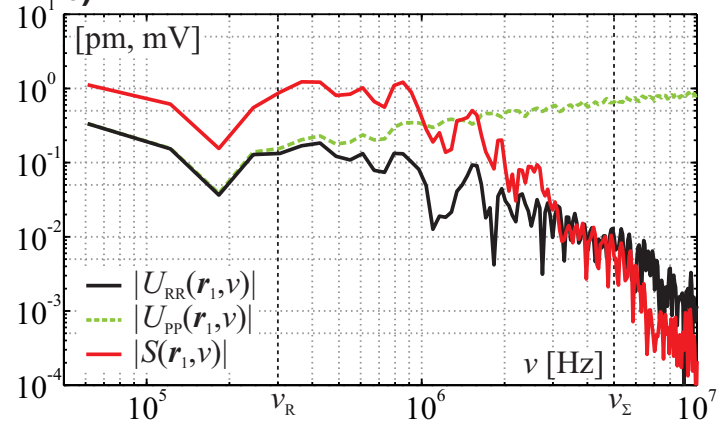

Fig. 5. a) A comparison of the two theoretically calculated sensor input waveform models and the sensor output voltage in sensor position 1 (same side as the source force) as well as b) a spectral comparison between the absolute values of their respective FFTs

Similarly, Fig. 6 shows all of these for the sensor in position 2 .

The distinctive peaks and abrupt changes in slope in the waveforms in Figs. 5a and 6a indicate wavearrivals.

The sensor transfer functions are calculated as described in section 1. The sensor input waveform models and the sensor output voltage measurements for both sensor positions are each fast Fourier transformed to obtain their spectra. The output measurement spectrum is then divided by the corresponding input waveform spectrum, as in Eq. (4), to obtain their sensor transfer functon spectrum.

Before the input models and the output measurements are fast Fourier transformed, they are all multiplied by the appropriate tapered cosine (Tukey) window function [22] to avoid any spurious frequencies in the FFTs [12] due to the possible 
artificial steps between the first and last points of the waveform models and the voltage measurements.

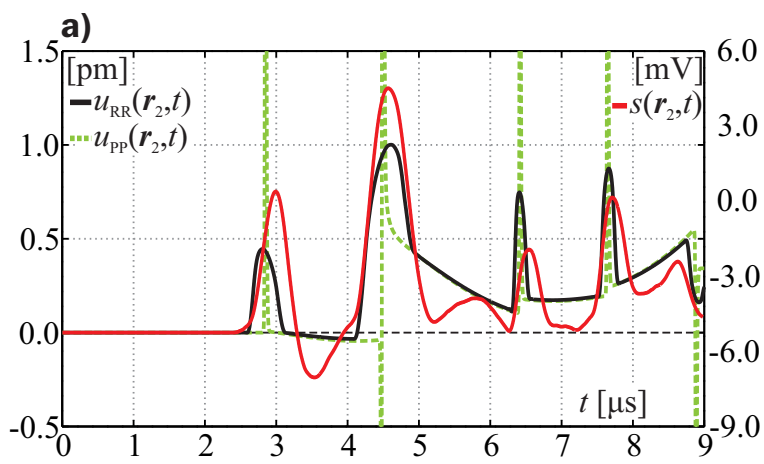

b)

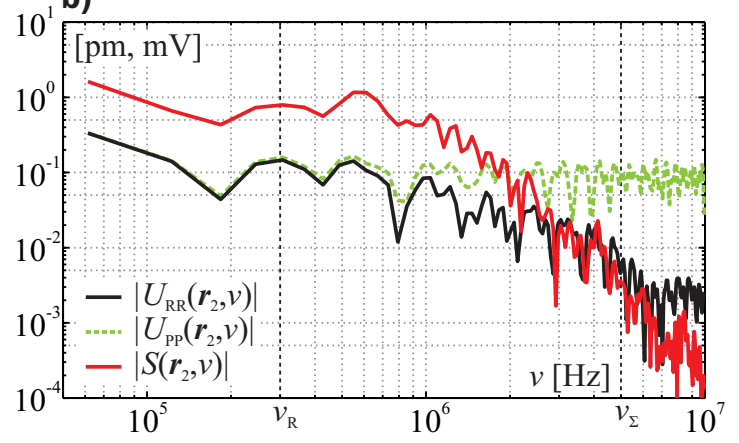

Fig. 6. a) A comparison of the two theoretically calculated sensor input waveform models and the sensor output voltage in sensor position 2 (opposite side as the source force) as well as b) a spectral comparison between the absolute values of their respective FFTs

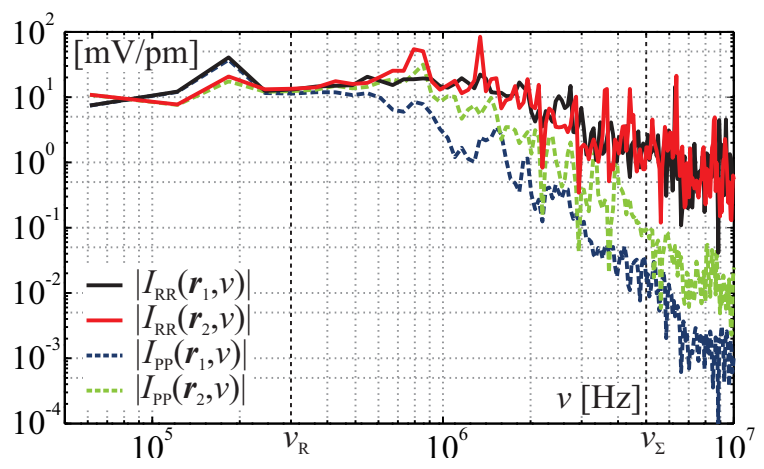

Fig. 7. A comparison of the absolute values of the calculated sensor transfer function spectra for real-source-real-sensor (RR) model and point-source-point-sensor (PP) model for both sensor positions

The absolute values of the sensor transfer function spectra for RR and PP models in both sensor positions are shown in Fig. 7 and are: $\left|I_{\mathrm{RR}}\left(\boldsymbol{r}_{1}, v\right)\right|,\left|I_{\mathrm{RR}}\left(\boldsymbol{r}_{2}, v\right)\right|$, $\left|I_{\mathrm{PP}}\left(\boldsymbol{r}_{1}, v\right)\right|,\left|I_{\mathrm{PP}}\left(\boldsymbol{r}_{2}, v\right)\right|$, respectively.

\section{DISCUSSION}

A comparison between the two theoretically calculated sensor input waveforms from the point-source-pointsensor and the real-source-real-sensor models and the sensor output voltage for both sensor positions shown in Figs. 5 and 6 shows notable differences.

Due to the unprecedented shortness of the ultrasound inducing laser pulse used here, the PP model waveform has very narrow spikes instead of the broader peaks that can be seen in the measured signals in Figs. 5a and 6a. It seems thus quite obvious that of the two models, the RR model produces a waveform that follows the contours of the measured signal much more closely than the hitherto used PP model. Most notably, although the measured sensor signal has a certain delay and a sort of 'inertia' compared to the RR model waveform, the duration and time distribution of the individual wave arrivals in the RR model correspond closely to the duration and time distribution of the wave arrivals in the PP model. Their respective FFT spectra show similar indications up to the sensor output noise levels at a frequency of about $v_{\Sigma}$ as well.

It is interesting to note that the PP model and RR model spectra for both sensor positions overlap at frequencies smaller than about $v_{\mathrm{R}}=300 \mathrm{kHz}$ and diverge greatly at frequencies greater than about $v \mathrm{R}$. This indicates that the source force distribution effect and the sensor aperture effect do not show at frequencies smaller than $v_{\mathrm{R}}$ while they are quite significant at frequencies greater than $v_{\mathrm{R}}$. The shortest waves at this frequency can be calculated to have wavelength of about $\lambda_{\mathrm{R}}=1.1 \mathrm{~cm}$, which is about an order of magnitude greater than the size of the source force impact area and the sensor contact area. For those area sizes, the PP model may apply for frequencies smaller than $v_{\mathrm{R}}$.

Furthermore, the calculated spectral sensor transfer functions in Fig. 7 also indicate the consistency of the RR model. The transfer functions of the RR model for both sensor positions seem to overlap reasonably, whereas the transfer functions of the PP model for each of the sensor positions do not. This can be further confirmed by calculating and comparing the absolute deviation $D$ between both of the RR model transfer function spectra $D_{\mathrm{RR}}=0.344$ and both of the PP model transfer function spectra $D_{\mathrm{PP}}=0.761$. From this it is evident that the spectra of the RR model deviate much less from each other than those of the PP model. Should the spectra be made smooth, the distinction in deviation for the RR 
model and the PP model would have been even more pronounced: $D_{\mathrm{RRsm}}=0.096$ and $D_{\mathrm{PPsm}}=0.788$.

It is also important to note that all of the corresponding sensor transfer functions start their divergence at a frequency of about $v_{\mathrm{R}}$ thus indicating again that the source force distribution effect and the sensor aperture effect start to manifest themselves at frequencies greater than $v_{\mathrm{R}}$.

The theory shows that these distinctions can mostly be attributed to the incorporation of the sensor aperture effect and the source force distribution effect in the RR model and their exclusion from the PP model, as explained in detail further on. The schematics of the incorporation or exclusion of the source force distribution effect and the sensor aperture effect for different waveform models are illustrated in Fig. 8.

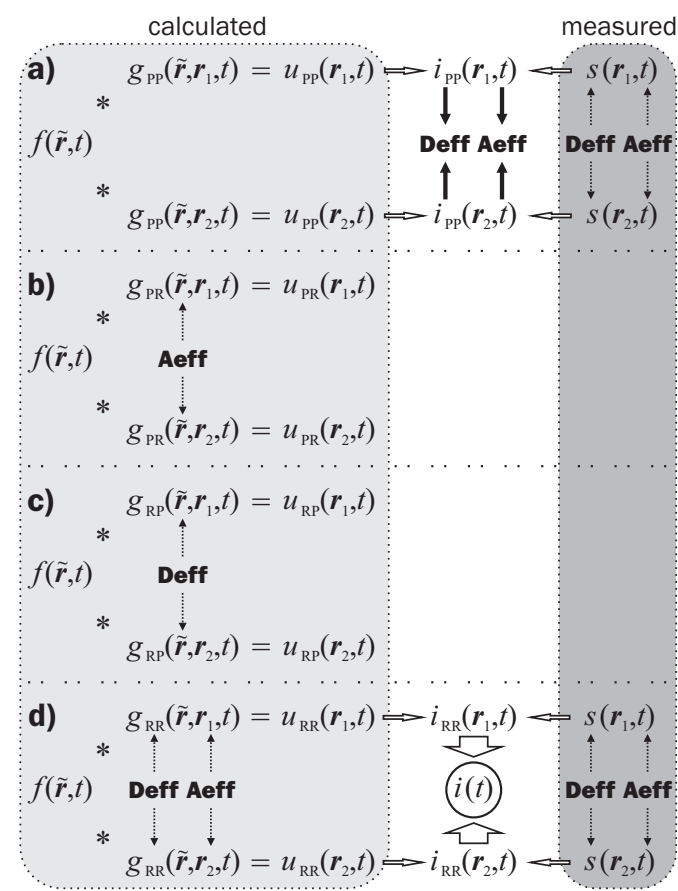

Fig. 8. Schematics of the mathematical incorporation of the source force distribution effect (Deff) and the sensor aperture effect (Aeff) for a) point-source-point-sensor (PP), b) point-sourcereal-sensor (PR), c) real-source-point-sensor (RP), and d) realsource-real-sensor (RR) models for both sensor positions as used and discussed here

It is worth noting that the aperture effect and the source force distribution effect are location specific, meaning that they are different at different relative positions of the source force area and the sensor contact area to each other.

Since the sensor outputs are responses to real waveform stimulations, these effects are always intrinsically incorporated into the measured sensor output signals $s\left(\boldsymbol{r}_{1}, t\right)$ and $s\left(\boldsymbol{r}_{2}, t\right)$. In the theoretically calculated models, however, this is not necessarily the case.

As Fig. 8a illustrates, the PP model does not incorporate any force distribution and sensor aperture effects while the sensor output signals naturally do. Therefore, when the sensor transfer functions for both sensor positions, $\left|I_{\mathrm{PP}}\left(\boldsymbol{r}_{1}, v\right)\right|$ and $\left|I_{\mathrm{PP}}\left(\boldsymbol{r}_{2}, v\right)\right|$, are derived from such a model, each still contains the source force and sensor aperture effects. Since these effects are specific for each sensor position, these transfer functions are position specific as well. This is the single most important reason for the mismatch of the PP sensor transfer functions in Fig. 7 at frequencies greater than $v_{R}$.

Conversely, as Fig. 8d illustrates, the RR model does incorporate the source force distribution effect and the sensor aperture effect. These effects are incorporated in the plate transfer functions $g_{\mathrm{RR}}\left(\tilde{\boldsymbol{r}}, \boldsymbol{r}_{1}, t\right)$ and $g_{\mathrm{RR}}\left(\tilde{\boldsymbol{r}}, \boldsymbol{r}_{2}, t\right)$, and subsequently in the sensor input waveform models $u_{\mathrm{RR}}\left(\boldsymbol{r}_{1}, t\right)$ and $u_{\mathrm{RR}}\left(\boldsymbol{r}_{2}, t\right)$. So, when the sensor transfer functions for both sensor positions, $\left|I_{\mathrm{RR}}\left(\boldsymbol{r}_{1}, v\right)\right|$ and $\left|I_{\mathrm{RR}}\left(\boldsymbol{r}_{2}, v\right)\right|$, are derived from this model they contain no such effects as the effects naturally incorporated into the measured sensor output signals are, in a way, sufficiently cancelled out by the same effects mathematically incorporated in the calculated sensor input waveforms at each sensor position. In this manner, the aperture effect and the source force distribution effect are properly accounted for in the RR model and thus eliminated from its sensor transfer functions. This is why, in Fig. 7, the RR transfer functions match quite well, even at frequencies greater than $v_{R}$, and a single, position independent sensor transfer function $|I(v)|$ can be obtained from them. It should be stressed that obtaining the proper, position independent sensor transfer function was the goal of this sensor calibration in the first place.

There exist two intermediate models between the two shown above. As Figs. 8b and 8c illustrate, the PR model and the RP model contain only the sensor aperture effect and the source force distribution effect, respectively. No sensor transfer functions are derived from them as they are only meant for the study of those effects further in the discussion.

If the use of the PP model in sensor calibration at higher frequencies were unavoidable, the best advice would be to calibrate the sensor in the position of its intended use, as the non-overlapping of the PP model sensor transfer functions clearly indicates their nonuniversality. 
All of this demonstrates the superiority of the RR model over the older PP model for high-frequency sensor calibration where the source force area and the sensor contact area are larger than the waveform wavelengths and the source force distribution effect and the sensor aperture effect become considerable.

The waveforms are in fact superpositions of many different kinds of waves each with different characteristics and each propagating with different velocities. For a quick estimate of the order of magnitude of their wavelengths, one can use the basic phase velocity equation: $\lambda=c / v$. The shortest and slowest reliably measured waves, at the noise limit frequencies of about $v_{\Sigma}$, have wavelengths of about $\lambda_{\min }=0.7 \mathrm{~mm}$ while the longest and fastest measured waves have wavelengths of several centimeters. It is difficult to measure these longer waves reliably as their arrivals span much of the measured time and only a few wavelengths are measured at best. Larger specimens would allow for longer measuring times and calibration at lower frequencies. The shortest measured waves are therefore much smaller than the sensor contact area and the longest are much longer than the source force impact area.

The presence of the sensor aperture effect can be clearly demonstrated using the point-source-realsensor model. A spectral comparison of the absolute values of the FFTs of the sensor input waveforms of the PP and PR models for both sensor positions is shown in Fig. 9a. It is worth highlighting the PR model for position 1 as it has distinctive lobes at certain frequencies. The minima between the lobes (some indicated by arrows in Fig. 9a) can be found to occur at frequencies close to:

$$
v_{\mathrm{S} n}=n \frac{c_{\mathrm{R}}}{2 r_{\mathrm{S} 0}}
$$

where $n$ is a positive integer and $c_{\mathrm{R}}$ is the propagation velocity of the Rayleigh waves, which are estimated to have dominant amplitudes on the top surface of the plate. It can thus be calculated: $v_{\mathrm{S} 1}=3.4 \mathrm{MHz}$, $v_{\mathrm{S} 2}=6.8 \mathrm{MHz}, v_{\mathrm{S} 3}=10.1 \mathrm{MHz}, v_{\mathrm{S} 6}=20.3 \mathrm{MHz}$, and so on. The reason for their slight shift towards higher frequencies in the measurements is probably due to the presence of other waves. This lobe phenomenom is due to the fact that surface waves propagate in the direction parallel to the sensor contact area. So, whenever the waveform wavelength is a multiple of the width of the sensor contact area, its signal decreases significantly as it is averaged out by the sensor. In position 2, the lobes are not visible in the spectrum, because the waves are incident at more acute angles.
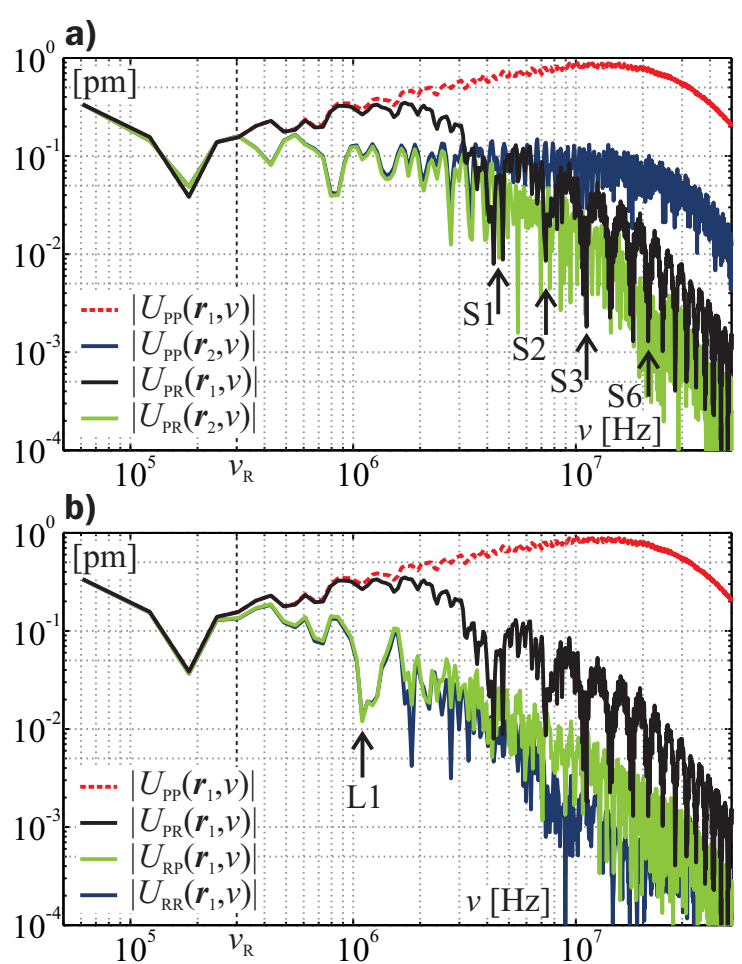

Fig. 9. A spectral comparison of the absolute values of the FFTs of the calculated sensor input waveform models a) for point-sourcepoint-sensor (PP) and point-source-real-sensor (PR) models for both sensor positions and b) for point-source-point-sensor (PP), point-source-real-sensor ( $P R)$, real-source-point-sensor $(R P)$ and

real-source-real-sensor (RR) models for sensor in position 1

The significance of the source force distribution effect can be demonstrated with the real-source-pointsensor model. A spectral comparison of the absolute values of the FFTs of the sensor input waveforms of all models for sensor in position 1 is shown in Fig. 9b. A pronounced minimum (indicated by an arrow in Fig. 9b), not present in the PP and PR models, is observed at a frequency of about $1 \mathrm{MHz}$. It can be explained as a manifestation of the source force distribution effect. Substituting $r_{\mathrm{S} 0}$ in Eq. (5) with the $r_{\mathrm{L} 0}$, one gets $v_{\mathrm{L} 1}=0.97 \mathrm{MHz}$. Here, the surface waves at this frequency are mostly canceled out by the geometry of the source force distribution relative to the sensor point position.

From Fig. 9b it can be observed that, while overlapping the lowest frequencies, the PP and the PR model spectra start diverging from the RP and the RR model spectra at a frequency of about $v_{\mathrm{R}}$. Furthermore, it can be observed that the former two continue to overlap until a frequency of about $1.0 \mathrm{MHz}$ while the latter two continue to overlap almost up to a frequency of 2.0 MHz. Even at higher frequencies, the RP model spectrum is much closer to the RR model spectrum 
than the other two and they have more similar contours as well.

In addition, it can also be observed that the one distinct lobe from the source force distribution effect is clearly present in the RR model spectrum, while none of the sensor aperture effect lobes are distinctly present in that spectrum. The sensor aperture effect is thus practically drowned out by the source force distribution effect in the RR model spectrum.

From this it can be concluded that the source force distribution effect has far greater presence in the RR model than the sensor aperture effect. This is mainly due to the fact that the source force impact area is significantly larger than the sensor contact area.

\section{CONCLUSIONS}

An absolute high-frequency calibration of a conical piezoelectric displacement sensor was presented here. The method uses a significantly improved theoretically calculated sensor input waveform model that incorporates the real dimensions of the source force area and the sensor contact area. In this manner, the real-source-real-sensor model enables the calculation of the proper sensor transfer function, which is devoid of the sensor aperture effect and the source force distribution effect and is therefore universal for any sensor position.

Experimentally, a Glaser-type piezoelectric displacement sensor has been calibrated on a glass plate with a high-reflectivity coating using a very short laser pulse reflection as the source force.

It was demonstrated that the critical role of incorporating the real dimensions of the source force area and the sensor contact area cannot be overlooked in the case of high-frequency ultrasound sensor calibration.

\section{AKNOWLEDGEMENTS}

The research was conducted as a part of the Optodynamics programme (P2-0392), in duration from 2015-1-1 to 2019-12-31, financed by the Slovenian Research Agency.

\section{REFERENCES}

[1] Požar, T., Možina, J. (2014). Detection of subnanometer ultrasonic displacements. Sattler, K.D. (Ed.). Fundamentals of Picoscience. CRC Press, Boca Raton [etc.], p. 553-577.

[2] Fortunko, C.M., Boltz, E.S. (1996). Comparison of absolute sensitivity limits of various ultrasonic and vibration transducers. Materials Science Forum, vol. 210-213, p. 471478, D0l:10.4028/www.scientific.net/MSF.210-213. 471.

[3] Grosse, C.U., Ohtsu, M. (Eds.). (2008). Acoustic Emission Testing: Basics for Research, Applications in Civil Engineering. Springer, Berlin. Dol:10.1007/978-3-540-69972-9.

[4] Sikorski, W. (Ed). (2012). Acoustic emission. InTech. DOI:10.5772/2070.

[5] Moore, P.O., Miller, R.K., Hill, E.v.K. (Eds.). (2005). Nondestructive Testing Handbook, 3rd ed. Vol. 6, Acoustic Emission Testing. American Society for Nondestructive Testing, Columbus.

[6] McLaskey, G.C., Thomas, A.M., Glaser, S.D., Nadeau, R.M. (2012). Fault healing promotes high-frequency earthquakes. Nature, vol. 491, no. 7422, p. 101-104. D0l:10.1038/ nature11512.

[7] Goodfellow, S.D., Young, R.P. (2014). A laboratory acoustic emission experiment under in situ conditions. Geophysical Research Letters, vol. 41, no. 10, p. 3422-3430. D0l:10.1002/2014GL059965.

[8] Royer, D., Dieulesaint, E. (2000). Elastic Waves in Solids II: Generation, Acousto-optic Interaction, Applications. SpringerVerlag, Berlin [etc.], Dol:10.1007/978-3-662-06938-7.

[9] Požar, T., Babnik, A., Možina, J. (2015). From laser ultrasonics to optical manipulation. Optics express, vol. 23, no. 6, p. 7978-7990, DOI:10.1364/0E.23.007978.

[10] Požar, T., Možina, J. (2013). Measurement of elastic waves induced by the reflection of light. Physical Review Letters, vol. 111, no. 18, 185501, D0l:10.1103/PhysRevLett.111.185501.

[11] Možina, J., Diaci, J. (2011). Recent advances in optodynamics. Applied Physics B: Lasers and Optics, vol. 105, no. 3, p. 557563, D0I:10.1007/s00340-011-4503-6.

[12] ASTM Standard E1106-86(2002)e1. Standard Method for Primary Calibration of Acoustic Emission Sensors. ASTM International, West Conshohocken.

[13] McLaskey, G.C., Glaser, S.D. (2012). Acoustic Emission Sensor Calibration for Absolute Source Measurements. Journal of Nondestructive Evaluation, vol. 31, no. 2, p. 157-168. Dol:10.1007/s10921-012-0131-2.

[14] McLaskey, G.C., Glaser, S.D. (2010). Hertzian impact: Experimental study of the force pulse and resulting stress waves. Journal of the Acoustical Society of America, vol. 123, no. 3, p. 1087-1096, D0I:10.1121/1.3466847.

[15] Cho, S.I., Lee, J.K., Lee, J.O., Jung, S.S., Lee, D.H., Seo, W.C. (2008). PZT Transducer Response to an Epicentral Acoustic Emission Signal During Glass Capillary Breakage. Journal of the Korean Physical Society, vol. 53, no. 6, p. 3213-3219. DOl:10.3938/jkps.53.3213.

[16] Goldsmith, W. (2001). Impact: The Theory and Physical Behaviour of Colliding Solids. Dover Publications, Mineola.

[17] Bosiger, G., Perhavec, T., Diaci, J. (2014). A Method for Optodynamic Characterization of Erbium Laser Ablation Using Piezoelectric Detection. Strojniški vestnik - Journal of Mechanical Engineering, vol. 60, no. 3, p. 172-178, D0l:10.5545/sv-jme.2013.1077.

[18] Hsu, N.N. (1985). Dynamic Green's functions of an infinite plate - a computer program. Technical Report No. NBSIR 853234, National Bureau of Standards, Washington. 
[19] KRN Services, Inc. (2015). Sensors, from: http://www. krnservices. com/sensors, accessed on 2015-06-01.

[20] Schneider, M., Berthe, L., Fabbro, R., Muller, M. (2008). Measurement of laser absorptivity for operating parameters characteristic of laser drilling regime. Journal of Physics $D$ : Applied Physics, vol. 41, no. 15, 155502. D0l:10.1088/0022 $3727 / 41 / 15 / 155502$.
[21] Weaver, J.H., Frederikse, H.P.R. (2009). Optical properties of selected elements. Lide, D.R. (Ed.), CRC Handbook of Chemistry and Physics, 89th ed. CRC Press, Boca Raton [etc.], p. 12-123-12-147.

[22] Bloomfield, P. (2000). Fourier Analysis of Time Series: An Introduction, $2^{\text {nd }}$ edition. John Wiley \& Sons, New York, D0I:10.1002/0471722235. 